\title{
Energetic Modes in Turbulent Pipe Flow From Resolvent Analysis
}

\author{
B. J. McKeon* \& A. S. Sharma ${ }^{\dagger}$
}

\begin{abstract}
We describe a method to investigate the mode shapes in turbulent pipe flow at a given wavenumber pair that are most responsive to harmonic forcing in the sense that the they correspond to the largest singular value in a Schmidt decomposition of the linear NavierStokes operator using the turbulent mean profile as the base flow. The ideas follow logically from the work of Sharma \& McKeon (2009), who considered a similar approach for laminar pipe flow.
\end{abstract}

\section{Introduction}

Predicting the structure of turbulent wall-bounded flows is a topic of intense fundamental and applied interest, yet, in general, understanding has advanced through experimental studies rather than theory due to the multi-scale nature of these flows. This is especially true at high Reynolds numbers, where for example recent work ${ }^{1-4}$ has revealed the previously undocumented contribution of very large streamwise scales to the variance of the streamwise velocity and turbulent energy production via the shear stress, both of which increase with increasing Reynolds number.

While linear transient growth processes have been used to correctly identify the spanwise wavelengths of the most energetic structures in turbulent channel $^{5}$ and boundary layer flows, ${ }^{6}$ the associated predicted streamwise wavelengths exceed the ranges that are observed in experiment and, indeed, even obtained in most facilities. A complete, predictive understanding of the scaling behavior of wall turbulence remains elusive.

In this manuscript we develop an analysis capable of predicting the harmonic modes that exhibit the largest response to forcing in turbulent pipe flow. This flow has the advantages of a well-defined geometry, streamwise homogeneity and linear stability characteristics. In addition, there are both high quality experimental data available for comparison, such as the mean velocity profiles as well as streamwise and wall normal turbulence statistics and spectra from the Princeton/ONR Superpipe, ${ }^{73,8}$ and other authors, ${ }^{1}$ as well as results from direct numerical simulation. ${ }^{9,10}$ The approach is similar in spirit to the analysis of Duggleby et al., who quantified the energy in propagating modes from an a posteriori Karhunen-Loeve (POD) analysis of full field information from a low Reynolds number DNS $\left(R e_{\tau}=180\right)$, with the advantage of being an a priori predictive tool.

We validate the efficacy of the simple, linear model, demonstrating that it is capable of capturing classical and empirically-observed features of wall turbulence.

\section{Approach}

In following analysis, we develop a formulation of the Navier-Stokes equations designed to examine the receptivity of turbulent pipe flow to forcing. In this way, we develop a framework that permits investigation of the form and likely magnitude of spatially- and temporally-harmonic, propagating finite-amplitude fluctuations about the turbulent mean profile in pipe flow.

*Graduate Aerospace Laboratories, California Institute of Technology, Pasadena, CA 91125, U.S.A.

${ }^{\dagger}$ Department of Aeronautics, Imperial College, London SW7 2AZ, United Kingdom 


\section{A. Pipe flow equations and non-dimensionalisation}

The non-dimensional Navier-Stokes equations for fully-developed, incompressible pipe flow with constant viscosity are given by

$$
\begin{aligned}
\partial_{t} \mathbf{u} & =-\nabla p-\mathbf{u} \cdot \nabla \mathbf{u}+\frac{1}{R e} \nabla^{2} \mathbf{u} \\
\nabla \cdot \mathbf{u} & =0
\end{aligned}
$$

The equations of motion are non-dimensionalised with respect to the pipe diameter and twice the bulk, volume-averaged velocity, $U_{\text {bulk }}$ (which in their study is equal to the laminar flow centreline velocity). Thus the Reynolds number in Equation 2 can be defined as

$$
R e=\frac{U_{b u l k} D}{\nu} .
$$

Here $D$ is the pipe diameter and $\nu$ is the kinematic viscosity. We retain the boundary layer terminology in fixing $y=1-r$, and $u, v\left(=-v^{\prime}\right)$ and $w$ as corresponding to the streamwise, wall-normal and azimuthal velocities such that $\mathbf{u}=\left(v^{\prime}, w, u\right)$.

\section{B. Model development}

We introduce a projection onto a divergence free basis: $\left\{\xi_{m}(r)\right\}$ in the radial direction, Fourier modes in the homogeneous spatial directions and the Laplace transform in time. Implicitly, we are considering a pipe that is infinitely long or periodic in the axial direction. Assuming fully developed flow allows us to express the velocity field as the sum of harmonic modes. Then,

$$
\begin{aligned}
u(r, x, \theta, t) & =\frac{1}{2 \pi i} \sum_{m, n} \int_{-i \infty}^{i \infty} \int_{-\infty}^{\infty} c_{m k n \omega} \xi_{m}(r) e^{i k x+i n \theta+s t} \mathrm{~d} k \mathrm{~d} s \\
& =\frac{1}{2 \pi i} \sum_{m, n} \int_{-i \infty}^{i \infty} \int_{-\infty}^{\infty} c_{m k n \omega} \xi_{m k n \omega}(r) \mathrm{d} k \mathrm{~d} s
\end{aligned}
$$

with $s=i \omega$, so that only harmonic forcing and response is considered.

The wavenumbers $(k, n, \omega)$ are non-dimensional such that $k=k^{\prime} R$, etc. The integration path for the Laplace transform is over the closed right half plane, which is analytic in the case of pipe flow. In the inviscid limit special treatment for singularities on the imaginary axis would be required, but we do not consider this limit. We work with the spatial $\mathcal{L}_{2}$ inner product throughout,

$$
(a, b)=\int_{x \in \Omega} a(x) b(x) d x .
$$

The basis functions are required to have the special properties

$$
\begin{aligned}
\left(\xi_{a}, \xi_{b}\right) & =\delta_{a b}, \\
\nabla \cdot \xi(r)_{m k n \omega} & =0 .
\end{aligned}
$$

This is done to eliminate the pressure term. For notational convenience we make the definitions

$$
\begin{aligned}
\mathbf{u}_{k n \omega} & =\left(\mathbf{u}, e^{i(\omega t+k x+n \theta)}\right) \\
\tilde{\mathbf{u}} & =\mathbf{u}-\mathbf{u}_{000} \\
\mathbf{f}_{k n \omega} & =\left(-\tilde{\mathbf{u}} \cdot \nabla \tilde{\mathbf{u}}, e^{i(\omega t+k x+n \theta)}\right) .
\end{aligned}
$$

This finally yields equations for the fluctuations that are linear in $\mathbf{u}_{k n \omega}$, and a base flow equation,

$$
\begin{aligned}
i \omega \mathbf{u}_{k n \omega} & =\mathcal{L}_{k n \omega} \mathbf{u}_{k n \omega}+\mathbf{f}_{k n \omega}, \quad \forall(k, n, \omega) \neq(0,0,0), \\
0 & =\mathbf{f}_{000}-\mathbf{u}_{000} \cdot \nabla \mathbf{u}_{000}+\frac{1}{R e} \nabla^{2} \mathbf{u}_{000} .
\end{aligned}
$$


The unknown constant forcing $\mathbf{f}_{000}$ describes the maintenance of $\mathbf{u}_{000}$ via the radial derivative of the Reynolds stresses, generated from interaction with the other modes. We can identify $\mathbf{u}_{000}$ with the turbulent mean velocity profile. Similarly, $\mathbf{f}_{k n \omega}$ describes the excitation of $\mathbf{u}_{k n \omega}$ by the triadic interaction with other wavenumbers. We cannot solve these equations without additional information because $\mathcal{L}_{k n \omega}$ incorporates $\mathbf{u}_{000}$ and $\mathbf{f}_{000}$ incorporates $\tilde{\mathbf{u}}$. However, the reader will notice that the perturbation equation (11) is a linear system with an unknown forcing $\mathbf{f}_{k n \omega}$. This fact is central to our treatment.

We avoid the closure problem for the base flow equation simply by knowing a priori the mean profile from experimental data. This allows calculation of $\mathcal{L}_{k n \omega}$ and precludes the need for an eddy viscosity formulation normally required. ${ }^{5,11}$

While $\mathbf{f}_{000}$ can be simply calculated from the mean profile, we do not know the Reynolds stress at any other wavenumber combination, $\mathbf{f}_{k n \omega}$. Our approach is not to make assumptions about this forcing, but to simply examine the response of (11) at individual $(k, n, \omega)$ triplets over the set of all possible harmonic forcings.

We expect downstream travelling waves such that $k$ and $\omega$ are of opposite sign: henceforth $k>0$ and $\omega \rightarrow-\omega$.

While Equation 11 is linear in $\mathbf{u}_{k n \omega}$, and $\mathcal{L}_{k n \omega}$ is identical to the operator obtained by linearising around the turbulent mean velocity profile, no linearisation has been performed. Nonlinear effects at other wavenumberfrequency combinations are retained through the action of the forcing $\mathbf{f}_{k n \omega}$. In physical terms, this forcing can be considered to stimulate fluctuations that may lead to a net energy gain because of the characteristics of $\mathcal{L}_{k n \omega}$.

Equation 11 can be rearranged as

$$
\mathbf{u}_{k n \omega}=\left(i \omega I-\mathcal{L}_{k n \omega}\right)^{-1} \mathbf{f}_{k n \omega} .
$$

The operator $\left(i \omega I-\mathcal{L}_{k n \omega}\right)^{-1}$ is called the resolvent and is the focus of our analysis. It provides a measure of the turbulent energy response that is possible for a given forcing.

\section{Most amplified modes}

We seek a decomposition of the resolvent at a particular wavenumber pair and frequency which ranks the response to forcing in some sense. We take the Schmidt decomposition (called the singular value decomposition in the discrete case) of the resolvent, namely

$$
(i \omega I-\mathcal{L})^{-1}=\sum_{j=1}^{\infty} \psi_{j}(k, n, y, \omega) \sigma_{j}(k, n, \omega) \phi_{j}^{*}(k, n, y, \omega)
$$

with an orthogonality condition

$$
\begin{aligned}
& \int_{y} \phi_{l}(k, n, y, \omega) \phi_{m}(k, n, y, \omega) \mathrm{d} y=\delta_{l m} \\
& \int_{y} \psi_{l}(k, n, y, \omega) \psi_{m}(k, n, y, \omega) \mathrm{d} y=\delta_{l m}
\end{aligned}
$$

and

$$
\sigma_{l} \geq \sigma_{l+1} \geq 0
$$

The $\phi_{j}$ and $\psi_{j}$ form the right and left Schmidt bases for the forcing and velocity fields and the real $\sigma_{j}$ are the singular values. This decomposition exists if there are no eigenvalues of $\mathcal{L}$ with zero real part and is unique up to a pre-multiplying complex factor on both bases of magnitude unity, corresponding to a phase shift of both forcing and response (in what follows each forcing and response pair are shown with zero relative temporal phase), and up to the ordering of the $\sigma_{j}{ }^{\text {'s. }}{ }^{12}$

This basis pair can then be used to decompose arbitrary forcing and the resulting velocity at a particular Fourier component

$$
\begin{array}{r}
f(k, y, n, \omega)=\sum_{l=1}^{\infty} \phi_{l}(k, n, y, \omega) a_{l}(k, n, \omega) \\
v(k, n, y, \omega)=\sum_{l=1}^{\infty} \sigma_{l}(k, n, \omega) \psi_{l}(k, n, y, \omega) a_{l}(k, n, \omega) .
\end{array}
$$


The energy of the same Fourier component of the resulting disturbance velocity is

$$
E(k, n, \omega)=\int_{y} v^{*}(k, n, y, \omega) v(k, n, y, \omega) \mathrm{d} y=\sum_{l=1}^{\infty} \sigma_{l}^{2}(k, n, \omega) a_{l}^{2}(k, n, \omega)
$$

Clearly the forcing shape that gives the largest energy at a particular frequency and wavenumber is given by $a_{l \neq 1}=0$. This approach permits the investigation of the dependence of maximum energy amplification on the form of the forcing in the wavenumber and frequency domain. The singular value decomposition for a given wavenumber pair and frequency corresponds to full volume, three component forcing and response modes ranked by the receptivity of the linear Navier-Stokes operator, which necessarily enforces zero (temporal) phase-shift between input and output. There is no requirement on the radial spatial phase, that is, the velocity response has the same $k$ and $n$ but not necessarily the same $y$ distribution as the forcing.

By Parseval's theorem, the energy integrated over frequency and wavenumber is equal to that integrated over the temporal and spatial domains (the spectral and physical spaces are isomorphic). As such, the 2-norm of the resolvent is the leading singular value, $\sigma_{1}$. This means that the normalised harmonic forcing that gives the largest disturbance energy in the $\mathcal{L}_{2}(\Omega \times[0, \infty))$ sense is $f=\phi_{1}$, with a 'gain' of $\sigma_{1}$. The next largest is $f=\phi_{2}$ and so on, at a particular wavenumber pair and frequency. The corresponding flow response modes are given by the related $v=\psi_{1}^{*}, \psi_{2}^{*}$, etc. For $\sigma_{j}$ near zero, the modes are not easily computed because they are effectively degenerate. However for large $\sigma_{j}$ the mode shapes are extremely robust to numerical error. We justify the use of this particular norm with the fact that the non-linear forcing is conservative in the perturbation energy. ${ }^{13}$

This decomposition permits analysis of what we call the particular forcing and response receptivity modes associated with large non-modal responses of a non-normal system. In this sense, this decomposition analyses the receptivity of the flow to forcing. In another sense, this decomposition examines the mode shapes associated with perturbations to the $\mathcal{L}$ operator (another pseudospectrum interpretation). Note that in the special case of a normal operator $\mathcal{L}$, the eigenvectors of the resolvent are coincident with the Schmidt bases found earlier.

\section{Computational approach}

The analysis of the linear operator, $\mathcal{L}$, was performed using a modified version of the pipe code of Meseguer \& Trefethen. ${ }^{14}$ For the particular problem under consideration here, the only modification to the linear operator is the use of the turbulent mean velocity profile instead of the steady, parabolic laminar base flow, as discussed above. We retain the same non-dimensionalization, namely using the centerline velocity for a laminar flow with the same mass-flux, the steady laminar flow pressure gradient and the pipe radius, $a$. This formulation is equivalent to using the bulk-averaged velocity, $\bar{U}$, and pipe diameter, $D$, as velocity and lengthscales, which is more natural to the turbulent problem. Of course, because the turbulent velocity profile is blunter than a laminar one, the constant mass-flux constraint means that the non-dimensional turbulent centerline velocity will always be less than one.

The form of the turbulent mean profiles was determined directly from experimental data obtained using Pitot probes in the Princeton/ONR Superpipe and reported by McKeon et al. ${ }^{7}$ This experimental data spans the Reynolds number range $31 \times 10^{3} \leq R e \leq 35 \times 10^{6}$ or $860 \leq R^{+}=R u_{\tau} / \nu \lesssim 5.35 \times 10^{5}$, where $u_{\tau}=\sqrt{\tau_{w} / \rho}$ is the friction velocity defined as the square root of the mean wall shear stress divided by the density. Interpolation between Reynolds numbers was performed in a process equivalent to assuming a Reynolds-number-independent form of the velocity profile. Issues of numerical stability limit our study to Reynolds numbers $R e \leq 10 \times 10^{6}$, well below the estimate of the Reynolds number at which the Superpipe results may show some effect of wall roughness $\left(R e \sim 20 \times 10^{6},{ }^{15}\right)$. Note that this Reynolds number range also spans conditions that have been described as representative of high Reynolds number turbulence in pipe flow by McKeon \& Morrison ${ }^{16}\left(R^{+} \gtrsim 5 \times 10^{3}\right)$ and in boundary layers by Hutchins \& Marusic ${ }^{17}\left(\delta^{+} \gtrsim 4 \times 10^{3}\right.$, where $\delta$ is the boundary layer thickness).

\section{E. A model of propagating modes}

The mode shapes investigated here are the response to harmonic forcing and can be combined in a linear sense because of their orthogonality, such that they should have a strong analogy to the spectral decomposition of 
spatial and temporal velocity fields from experiments and simulations. Their wave-like nature implies that the component of normal velocity of each mode in the streamwise direction, $U_{p}$, is given by $U_{p}=\omega / k$. In global terms, we expect that this analysis will give some insight into the turbulent energy spectrum.

\section{Results}

A concatenation of experimental results in wall turbulence in general, and specifically in pipe flow have shown that a streamwise wavenumber $k=1$ is representative of the very large scale motion (VLSM) phenomenon in a range of wall-bounded flows, ${ }^{3,4,16,18,19}$ although the recent study of Monty et al. ${ }^{20}$ suggests that the exact details vary from flow to flow. The appropriate spanwise wavenumber is less clear: spanwise measurements in pipe flow ${ }^{19}$ suggest $\lambda_{z} \approx 0.5 R(n \sim 12)$, while the POD and correlations ${ }^{21}$ indicate that lower values, $n \sim 3$, are more realistic for the VLSMs. We investigate $n=10$ as a compromise that follows the expected critical layer aspect ratio and is in reasonable agreement with the work of both Monty and Bailey. For this mode, the first singular value is orders of magnitude larger than the second one, such that we expect the first velocity mode to be dominant in our observations. In addition, the growth of the first singular value with increasing Reynolds number is slow (figure 1).

The variation of mode shape for $(k, n, \omega)=(1,10,0.67)$ is shown in figure 2 for $R e=75 \times 10^{3}$. Earlier work ${ }^{22}$ has shown that this wavenumber/frequency combination corresponds to a special mode that is both attached to the wall and critical, explicitly where the streamwise wavespeed reaches the local mean velocity, over a range of Reynolds numbers. Clearly the largest response is in the axial velocity component, with significantly smaller energetic content in the wall-normal and azimuthal components. Note that the azimuthal velocity has a similar wall-normal distribution to the axial velocity, suggestive of quasi-streamwise vorticity with a small inclination to the wall. The wall-normal velocity, while small, shows no spatial phase variation in the wall-normal direction.

Of course the modes that would be observed in practice would depend on the product of the response and the initial forcing amplitude. However there is excellent agreement between the mode shown in figure 2 and the shape of the most amplified propagating POD modes in the work of Duggleby et al. . Notably, for modes that are very long in the streamwise direction compared to their azimuthal width, the mode shapes appear "roll-like" with an associated kinetic energy that is concentrated in the streamwise fluctuations, small contribution from the azimuthal component and virtually no energy in the wall-normal velocity. The shape observed in the response mode of figure 2 is also strongly reminiscent of recent observations and inferences of the wall normal variation of the very large scale motions (VLSM) in channel ${ }^{23}$ and turbulent boundary layer ${ }^{24}$ flows. Thus we conclude that the simple model of the preceding section is capable of predicting the velocity distribution associated with the very large scale motion in pipe flows.

The fourth panel of figure 2 shows the distribution of Reynolds stress, $-u v$. Clearly the contribution from this mode is concentrated close to the wall and takes both signs as the distance from the wall is increased. The trend is consistent with the results of Guala et al. ${ }^{25}$ who showed that the very large scales are active in terms of carrying Reynolds stress, with a peak further from the wall than the location of the mean value.

The forcing distribution giving rise to this response, shown in figure 3, is predominantly in the wall-normal and azimuthal components, as would be expected from previous studies that have predicted the maximum response to occur for streamwise constant disturbances with $k=0$, with concurrent cross-stream forcing. For reference, the velocity response for even longer structures, with $k=0.0001 \rightarrow 0$ and $(n, \omega)=(10,0.67 k)$ is shown in figure 4 , and can be seen to represent the familiar rolls and streaks that fill the pipe cross-section. We can interpret the VLSM mode as representative of one of the longest modes that can be sustained in pipe flow. Streamwise velocity spectra show that there is an energy peak associated with this wavenumber. Comparison of figures 2 and 4 show that the effect of increasing the wavenumber with $R e, n$ and $\omega$ held constant is to tilt the rolls and streaks and confine them to a region close to the wall.

For this VLSM mode and perhaps for the more general class of modes that fall in this transitional, or critical, category, the appropriate scaling velocity is the centerline velocity, rather than the friction velocity which is a scale associated with the wall region and imposed on the outer flow through the boundary conditions. This was proposed by Jimenez et al. ${ }^{26}$ who observed that the so-called "global" modes in channel flow simulations appeared to convect with a velocity equal to $0.5 U_{C L}$.

Based on this scaling with two-thirds of the centerline velocity, the location of the energy peak associated with 
this VLSM-like mode can be predicted using the logarithmic profile for the mean velocity and the necessary condition for this scaling that the outer/core flow exhibits Reynolds similarity such that the inner-scaled deviation of the centerline velocity from a log law is a constant, $C$, i.e.

$$
\begin{gathered}
U^{+}\left(y^{+}\right)=\frac{1}{\kappa} \ln y^{+}+B \\
U_{C L}^{+}=U^{+}\left(y^{+}=R^{+}\right)=\frac{1}{\kappa} \ln R^{+}+B+C .
\end{gathered}
$$

The velocity reaches a value of two-thirds the centerline velocity at an inner-scaled wall-normal distance $y_{2 / 3}^{+}$ that can be predicted as follows:

$$
U^{+}=\frac{1}{\kappa} \ln y_{2 / 3}^{+}+B=\frac{2}{3}\left(\frac{1}{\kappa} \ln R^{+}+B+C\right),
$$

or, rearranging,

$$
y_{2 / 3}^{+}=a R^{+2 / 3}
$$

where $a=\exp \left(\frac{\kappa}{3}(2 C-B)\right)$. For a pipe $\kappa=0.421, B=5.60^{7}$ and $C \approx 2,{ }^{27}$ so $a=0.8$. Equation 20 represents a non-observational attempt to predict the location of the peak in the streamwise energy associated with the VLSMs, and the agreement with both the $(k, n)=(1,10)$ mode peak and the previously-published experimental variation shown in figure 5 can be seen to be excellent. For the experimental data the location of the VLSM peak at each Reynolds number was determined by considering the low wavenumber spectral peak in the hot-wire dataset of Morrison et al. ${ }^{3}$ as reported by McKeon. ${ }^{28}$ The error bars on the peak position shown in the figure are conservative and correspond to the next closest wall-normal locations at which spectra were obtained. While probe resolution effects may be a concern at the highest Reynolds number, the data at $R^{+}=19 \times 10^{3}$ clearly represent an outlier.

\section{Conclusions}

We have shown that a simple model with unstructured forcing applied to the dominant mode at a wavenumberfrequency combination representative of the very large scale motions in turbulent pipe flow reproduces several experimentally-observed features of those structures, including the componentwise velocity distribution and the wall-normal location of the peak in the streamwise velocity fluctuations. Work is ongoing to extend this analysis to other modes.

The support of NSF CAREER award grant number 0747672 (program managers William Schultz and H. Henning Winter), Air Force Office of Scientific Research (program manager John Schmisseur), grant number FA9550-09-1-0701(B.J.M.), an Imperial College Junior Research Fellowship and the EPSRC (A.S.) is gratefully acknowledged. We would also like to thank A. Meseguer and L. Trefethen for publishing their pipe code.

\section{References}

\footnotetext{
${ }^{1}$ Adrian, R. J., "Vortex organization in wall turbulence," Phys. Fluids, Vol. 19, No. 041301, 2007.

${ }^{2}$ Kim, J. and Lim, J., "A linear process in wall-bounded turbulent shear flows," Phys. Fluids, Vol. 12, No. 8, 1993, pp. 18851888.

${ }^{3}$ Morrison, J. F., Jiang, W., McKeon, B. J., and Smits, A. J., "Scaling of the streamwise velocity component in turbulent pipe flow," J. Fluid Mech., Vol. 508, 2004, pp. 99-131.

${ }^{4}$ Hutchins, N. and Marusic, I., "Evidence of very long meandering features in the logarithmic region of turbulent boundary layers," J. Fluid Mech., Vol. 579, 2007, pp. 1-28.

${ }^{5}$ del Álamo, J. C. and Jiménez, J., "Linear energy amplification in turbulent channels," J. Fluid Mech., Vol. 559, 2006, pp. 205-213.

${ }^{6}$ Cossu, C., Pujals, G., and Depardon, S., "Optimal transient growth and very large scale structures in turbulent boundary layers," J. Fluid Mech., Vol. 619, 2009, pp. 79-94.

${ }^{7}$ McKeon, B. J., Li, J., Jiang, W., Morrison, J. F., and Smits, A. J., "Further observations on the mean velocity distribution in fully developed pipe flow," J. Fluid Mech., Vol. 501, 2004, pp. 135-147.

${ }^{8}$ Zhao, R. and Smits, A. J., "Scaling of the wall-normal turbulence component in high-Reynolds-number pipe flow," J. Fluid Mech., Vol. 576, 2007, pp. 457-473.
} 
${ }^{9}$ Duggleby, A., Ball, K. S., Pail, M. R., and Fischer, P. F., "Dynamical eigenfunction decomposition of turbulent pipe flow," J. Turbulence, Vol. 8, No. 43, 2007, pp. 1-24.

${ }^{10} \mathrm{Wu}, \mathrm{X}$. and Moin, P., "A direct numerical simulation study on the mean velocity characteristics in pipe flow," J. Fluid Mech., Vol. 608, 2008, pp. 81-112.

${ }^{11}$ Reynolds, W. C. and Hussain, A. K. M. F., "The mechanics of an organized wave in shear flow. Part 3. Theoretical models and comparisons with experiment." J. Fluid Mech., Vol. 54, 1972, pp. 263-288.

${ }^{12}$ Young, N., An Introduction to Hilbert Space, Cambridge University Press, Cambridge, UK, 1988.

${ }^{13}$ Sharma, A. S., McKeon, B. J., Morrison, J. F., and Limebeer, D. J. N., "Stabilising control laws for the incompressible Navier-Stokes equations using sector stability theory," 3rd AIAA Flow Control Conference, Vol. AIAA-2006-3695, June 2006. ${ }^{14}$ Meseguer, A. and Trefethen, L. N., "Linearized pipe flow to Reynolds number 107," J. Comp. Phys., Vol. 186, 2003, pp. 178197.

${ }^{15}$ Shockling, M. A., Allen, J. J., and Smits, A. J., "Effects of machined surface roughness on high-Reynolds-number turbulent pipe flow," J. Fluid Mech., Vol. 564, 2006, pp. 267-285.

${ }^{16}$ McKeon, B. J. and Morrison, J. F., "Asymptotic scaling in turbulent pipe flow," Phil. Trans. Royal Soc. A, Vol. 365, 2007, pp. $771-787$.

${ }^{17}$ Hutchins, N. and Marusic, I., "Large-scale influences in near-wall turbulence," Phil. Trans. Royal Soc. A, Vol. 365, 2007, pp. 647-664.

${ }^{18}$ Kim, K. C. and Adrian, R. J., "Very large-scale motion in the outer layer," Phys. Fluids, Vol. 11, 1999, pp. 417-422.

${ }^{19}$ Monty, J. P., Stewart, J. A., Williams, R. C., and Chong, M. S., "Large-scale features in turbulent pipe and channel flows," J. Fluid Mech., Vol. 589, 2007, pp. 147-156.

${ }^{20}$ Monty, J. P., Hutchins, N., Ng, H. C. H., Marusic, I., and Chong, M. S., "A comparison of turbulent pipe, channel and boundary layer flows," J. Fluid Mech., Vol. 632, 2009, pp. 431-442.

${ }^{21}$ Bailey, S. C. C., Hultmark, M., Smits, A. J., and Schultz, M. P., "Azimuthal structure of turbulence in high Reynolds number pipe flow," J. Fluid Mech., Vol. 615, 2008, pp. 121-138.

${ }^{22}$ McKeon, B. J. and Sharma, A., "A critical layer model for turbulent pipe flow," (submitted), 2009.

${ }^{23}$ Chung, D. and McKeon, B. J., "Large-eddy simulation investigation of large-scale structures in a long channel flow," (Submitted), 2009.

${ }^{24}$ Guala, M., Metzger, M. J., and McKeon, B. J., "Interactions within the turbulent boundary layer at high Reynolds number," (submitted), 2009.

${ }^{25}$ Guala, M., Hommema, S. E., and Adrian, R. J., "Large-scale and very-large-scale motions in turbulent pipe flow," J. Fluid Mech., Vol. 554, 2006, pp. 521-542.

${ }^{26}$ Jiménez, J., del Álamo, J. C., and Flores, O., "The large-scale dynamics of near-wall turbulence," J. Fluid Mech., Vol. 505, 2004, pp. 179-199.

${ }^{27}$ McKeon, B. J., Zagarola, M. V., and Smits, A. J., "A new friction factor relationship for fully developed pipe flow," J. Fluid Mech., Vol. 538, 2005, pp. 429-443.

${ }^{28}$ McKeon, B. J., "Scaling in wall turbulence: scale separation and interaction," AIAA Paper, Vol. 2008-4237, 2008.

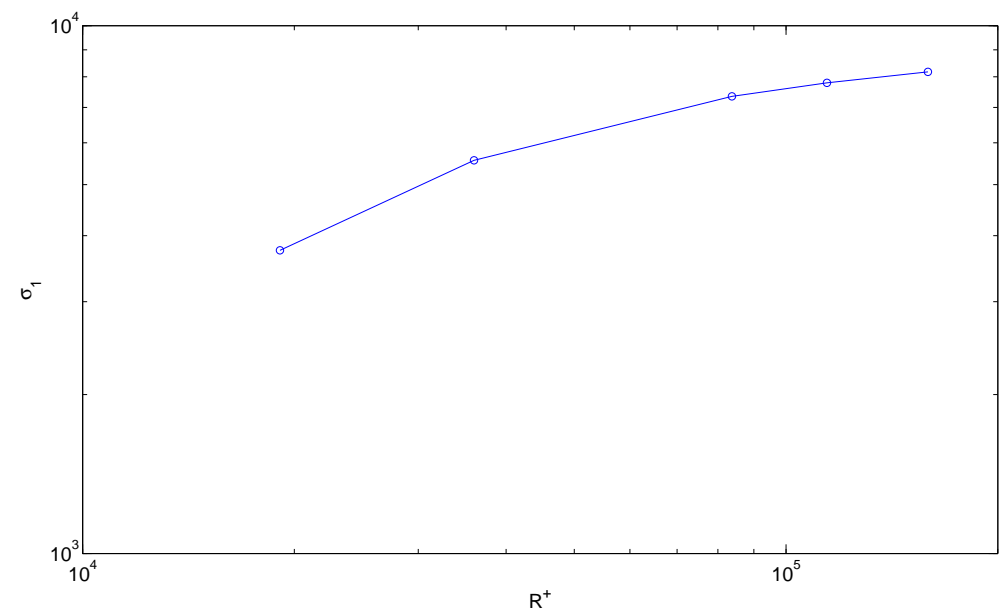

Figure 1. The variation of the first singular value, $\sigma_{1}$, for a mode with $(k, n, \omega)=(1,10,0.4)$. 

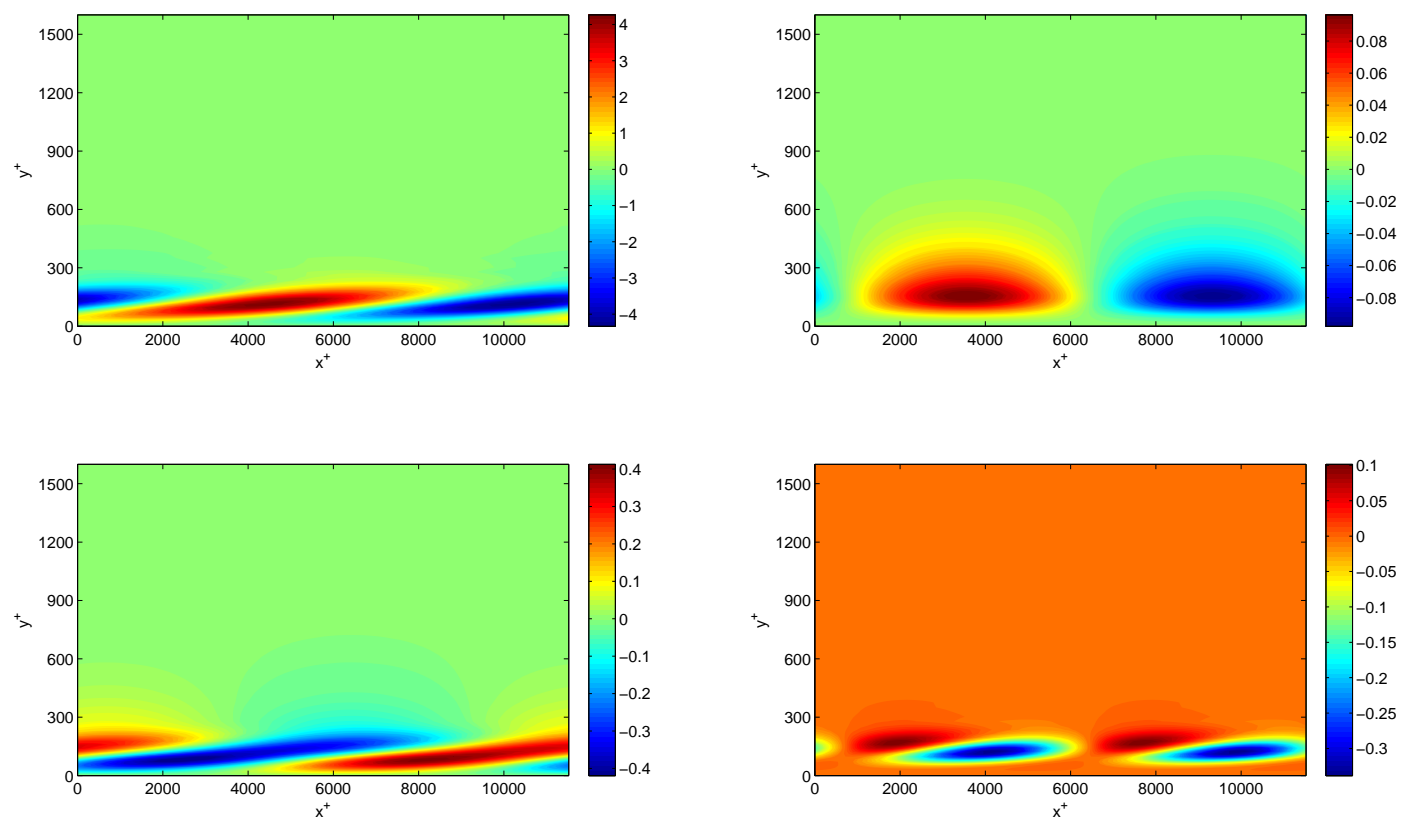

Figure 2. Streamwise cross-sections of the pipe showing the componentwise spatial distribution of turbulent energy at $R e=75 e 3$. From left to right, top to bottom: streamwise, radial and azimuthal components normalized on their maximum values, Reynolds stress - uv. Helical mode with $(k, n, \omega)=(1,10,0.4)$.
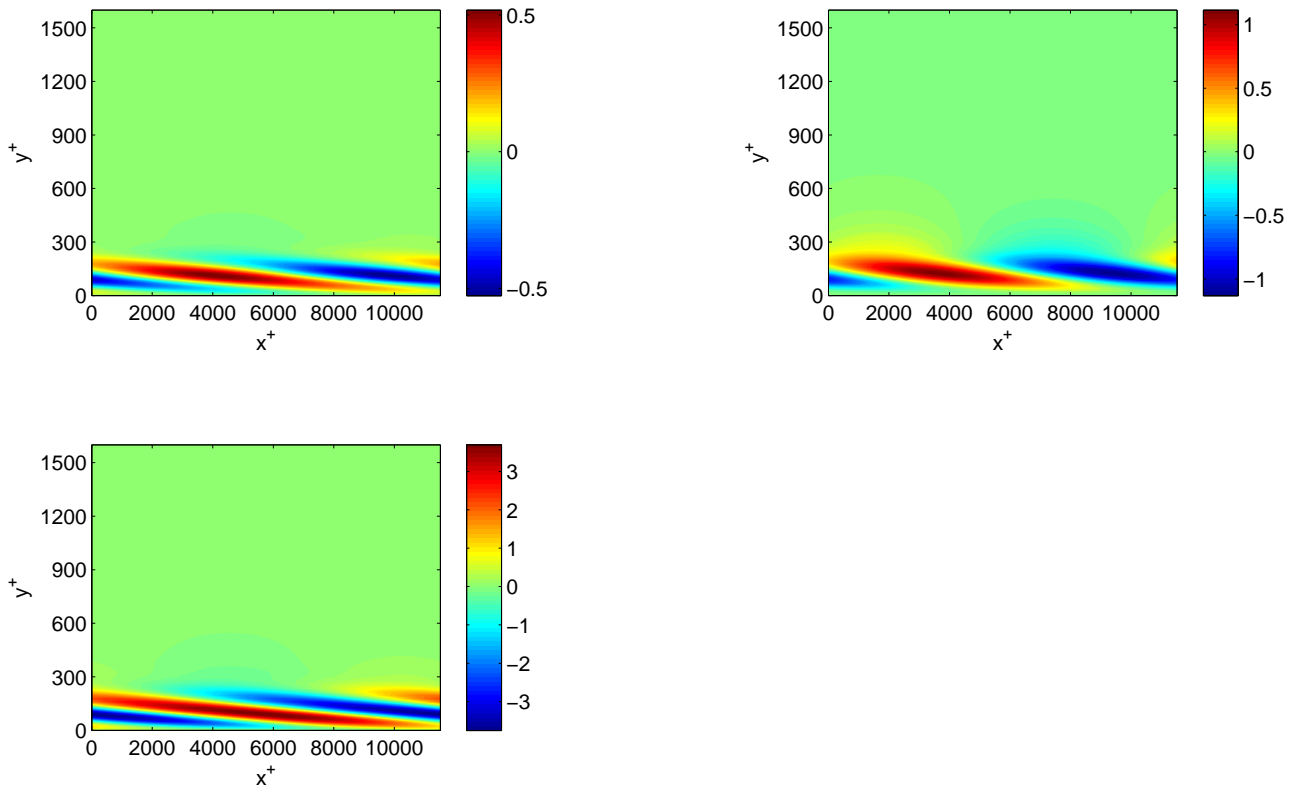

Figure 3. First forcing mode giving rise to the velocity response in figure 2, i.e. $(k, n, \omega)=(1,10,0.4)$ and $R e=75 \times 10^{3}$. From left to right, top to bottom: streamwise, radial and azimuthal components normalized on their maximum values. 

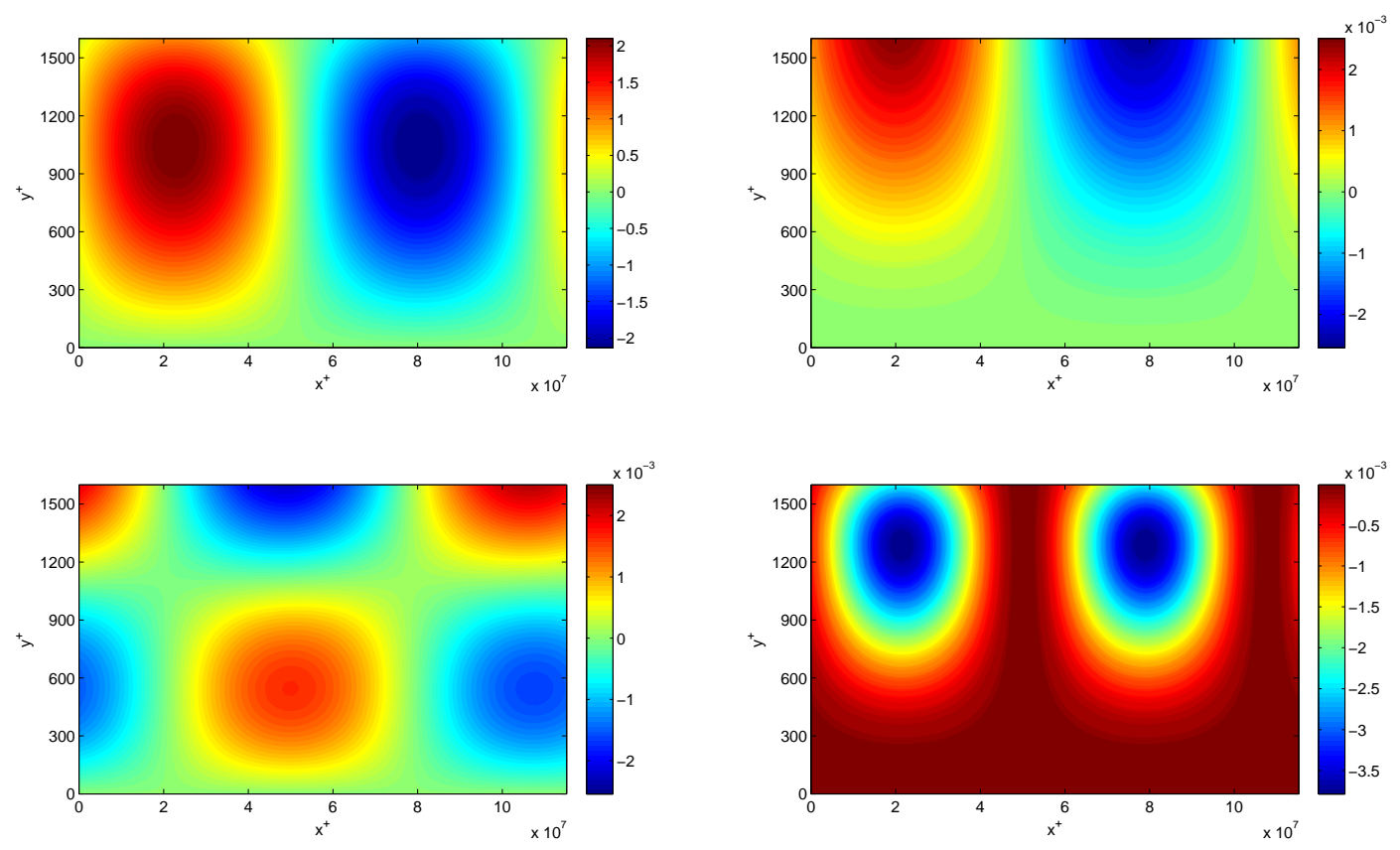

Figure 4. Streamwise cross-sections of the pipe showing the componentwise spatial distribution of turbulent energy at $R e=75 e 3$. From left to right, top to bottom: streamwise, radial and azimuthal components normalized on their maximum values, Reynolds stress - uv. Helical mode with $(k, n, \omega)=(0.0001,10,0.00004)$.

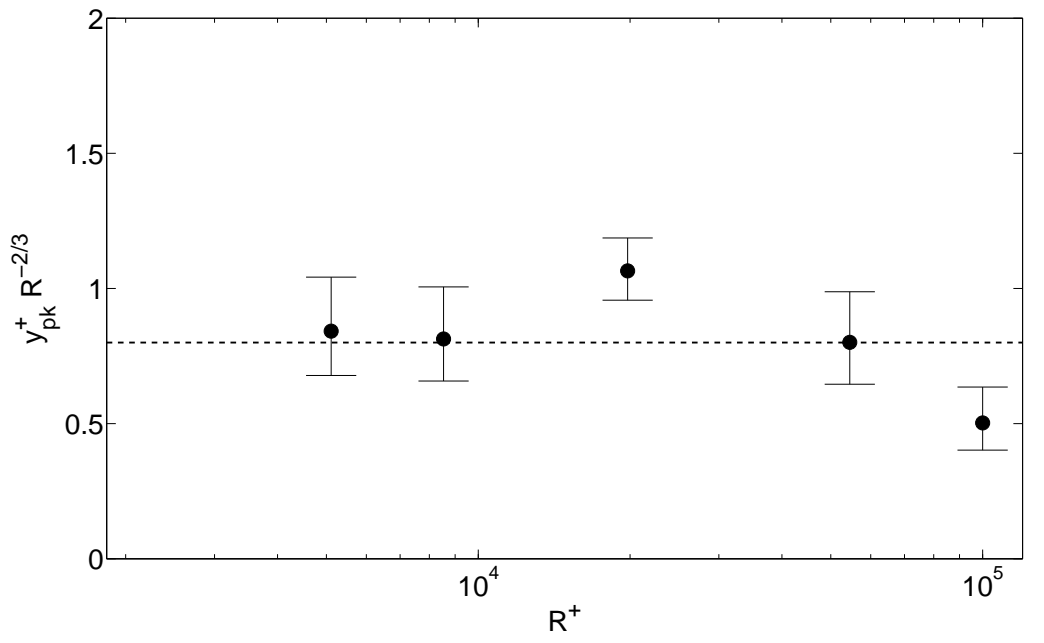

Figure 5. Comparison of experimental pipe flow results on the $y^{+}$-location of the spectral peak associated with the VLSM modes and the prediction of equation 20. •: Superpipe data (Morrison et al., 2004); dashed line: $y^{+}=0.8 R^{+2 / 3}$. Note that the highest Reynolds number data point is likely affected by hot-wire spatial resolution (the non-dimensional wire length in this case is $l^{+}=385$ ). 